\title{
Das potenzielle photographische Bild
}

\author{
STEFFEN SiEgEL
}

In den medientheoretischen Debatten um die Referenzialität der Photographie stand vorderhand das im Bild Sichtbare als ein "Bruchstück der Wirklichkeit" im Zentrum der Aufmerksamkeit. Die hierbei gestellten Fragen nach den Optionen von Einschluss und Ausschluss, von Detail und Ganzem lassen sich, wie in diesem Artikel gezeigt werden soll, jedoch nur dann sinnvoll diskutieren, wenn als dritte Kategorie Bildtechniken des visuellen Anschlusses in den Blick genommen werden. Denn von entscheidender Bedeutung für eine Ästhetik des photographischen Bildes ist sein Jenseits, das durch Rahmen und Ränder nicht abgeschnitten, sondern potenziell sichtbar gehalten wird. Anhand jüngerer photographischer Arbeiten der Künstler David Hockney, Jan Wenzel und Peter Hendricks wird dieses latente Verhältnis von Sichtbarkeit und Unsichtbarkeit, Präsenz und Absenz betrachtet und als eine Ästhetik potenzieller photographischer Bildlichkeit näher bestimmt.

Für Julia Staffel, nicht nur, aber auch wegen des Pearblossom Highway.

I

Photographien besitzen ein besonderes Verhältnis zu der sie umgebenden Wirklichkeit. Diese These gehört zum basalen Repertoire der Theoriegeschichte des photographischen Bildes und ist bereits in jenen Jahren erstmals formuliert worden, als diese Technik selbst gerade eben erst erfunden worden war. Denn bereits William Henry Fox Talbot dachte in seinem Pencil of Nature nicht allein über die verblüffende Präzision der neuen Darstellungsmöglichkeiten nach; vielmehr verband er mit der Photographie »eine neue Art von Beweis, wenn die stumme Zeugenschaft des Bildes vor Gericht " ${ }^{1}$ etwa gegen einen hypothetischen Dieb der von Talbot photographierten wertvollen Porzellan- und Glaswaren aussagen würde. Das photographische Bild, heißt dies, kann den Beweis für eine Existenz führen,

1 William Henry Fox Talbot: Der Zeichenstift der Natur [1844-1846]. In: Wilfried Wiegand (Hg.): Die Wahrheit der Photographie. Klassische Bekenntnisse zu einer neuen Kunst, Frankfurt am Main 1981, S. 45-89; hier S. 62. 
die außerhalb von ihm liegt. Es ist diese Idee einer spezifischen 'Zeugenschaft des Bildes` (`testimony of the picture`), die für jedes weitere Nachdenken über den juridischen, den ethischen und insbesondere den ontologischen Status des photographischen Bildes eine der leitenden Metaphern bleiben wird. ${ }^{2}$ Doch selbst wenn Michelangelo Antonioni in geradezu emblematischer Weise mit seinem Film Blow $U_{p}$ unterstrichen hat, wie flüchtig, unzuverlässig und prekär das Verhältnis zwischen photographischem Bild und Wirklichkeit bei - ganz wörtlich verstanden näherem Hinsehen ist, so wird, selbst im Kontext eines Nachdenkens über postmoderne Konzeptkunst, die Verpflichtung der Photographie auf eine Abbildfunktion noch immer mit ihrer besonderen "physischen Natur « ${ }^{3}$ begründet.

Der Akzent dieser Fragestellung nach dem besonderen Wirklichkeitsbezug des photographischen Bildes lässt sich indes auch anders setzen. Denn abseits des ohnehin voraussetzungsreichen - Begriffs /Wirklichkeit ist es die an das photographische Bild gerichtete Idee einer `Bezugnahme`, die nähere Betrachtung verdient. In welcher Weise sich Photographien mit dem auf ihnen Gezeigten überhaupt auf etwas beziehen, das ist weit weniger selbstverständlich, als die kurrente Formel ‘Ça a été glauben machen will. Es steht nicht allein in Frage, was dieses ‘ça‘, von dem Roland Barthes so emphatisch schrieb, eigentlich ist, sondern auch, wie es durch das im Bild Sichtbare überhaupt erreicht werden kann. Bereits früh wurde mit der Rede vom "Silberspiegel ${ }^{4}$ jedenfalls ein Leitmotiv photographischer Referenzialität intoniert, das, wie es scheint, unter den sich verändernden technischen Bedingungen des Mediums einzig umformuliert, jedoch nie grundsätzlich in Frage gestellt werden musste. Vollends zu seinem Recht scheint der von Barthes gewählte Indikativ (‘a été ‘) aber spätestens dann zu kommen, wenn der Wirklichkeitsbezug des Bildes nicht allein als eine Referenz, sondern vielmehr als Präsenz gedacht wird, die sich im Bild einnistet. Selbst wenn dieses in der Tat höchst "untote Paradigma " ${ }^{5}$ der Indexikalität längst und mit mehr als einem guten

2 Bernd Stiegler: Zeuge. In: ders.: Bilder der Photographie. Ein Album photographischer Metaphern, Frankfurt am Main 2006, S. 264-268.

3 Jeff Wall: Zeichen der Indifferenz: Aspekte der Photographie in der, oder als, Konzeptkunst [1995]. In: ders.: Szenarien im Bildraum der Wirklichkeit. Essays und Interviews, hg. von Gregor Stemmrich, Amsterdam, Dresden 1997, S. 375-434; hier S. 377.

4 Stiegler 2006 (wie Anm. 2), S. 201-203. Siehe hierzu insbesondere aber auch Philippe Dubois: Der fotografische Akt. Versuch über ein theoretisches Dispositiv, [Paris 1990], übers. von Dieter Hornig, Amsterdam, Dresden 1998, S. 31-40. In vergleichbarer Weise betont überdies die ältere, schon für die (Bildnis-)Malerei kurrente Formel `Nach dem Leben diesen Aspekt der Referenzialität. Mit Blick auf die Photographie siehe hierzu Wolfgang Brückle: Nach dem Leben. In: Ute Eskildsen (Hg.): Ein Bilderbuch. Fotografische Sammlung im Museum Folkwang, Göttingen 2003, S. 11-17.

5 Peter Geimer: Das Bild als Spur. Mutmaßungen über ein untotes Paradigma. In: Sybille Krämer, Werner Kogge, Gernot Grube (Hg.): Spur. Spurenlesen als Orientierungstechnik und Wissenskunst, Frankfurt am Main 2007, S. 95-120. Siehe außerdem Dubois 1998 (wie Anm. 4), S. 49-57. Stiegler 2006 (wie Anm. 2), S. 216-219. 
Grund nachdrücklich kritisiert worden ist, die mit seiner Formulierung sich abzeichnende und seit dem Pencil of Nature in vielfachen Variationen an das photographische Bild gerichtete Erwartung eines besonderen Verhältnisses zwischen Medium und Wirklichkeit kann als theoretische Modellierung einer Beziehung von Nähe und Authentizität ernst genommen werden.

Gewiss kein Zufall ist es daher, wenn bereits im Titel der ersten eingehenden Auseinandersetzung mit der digitalen Photographie die Frage der Wahrheitsfähigkeit dieses Mediums reformuliert worden ist und - durch die Ausrufung einer 'post-photographischen Ära - als erledigt und abgeschlossen angesehen wurde. ${ }^{6}$ Tatsächlich scheint es einer Rhetorik des Neuen geschuldet, dass der aufziehenden "digitalen Weltordnung" zugestanden wird, »unter sich die analogen Verhältnisse [zu] begraben «. ${ }^{7}$ Doch ist hierbei weniger die Frage nach der Richtigkeit einer solchen Prognose von Interesse, als vielmehr die Tatsache, dass , Wahrheit und photographische Bildlichkeit überhaupt, und sei es auch nur spekulativ, in einen so engen Zusammenhang gerückt werden. In einem kurzen Nachsatz zu seinem Pencil of Nature hatte Talbot versichert, dass alle Tafeln "allein durch die Einwirkung des Lichtes hervorgerufen worden [sind], ohne irgendeine Mithilfe von Künstlerhand. ${ }^{8}$ Dieser Satz ist gewiss auch deshalb seither so oft zitiert worden, da er einen erstaunlichen, wenn nicht sogar paradoxen Akt der Selbstverleugnung durch einen der ersten Photographen-Künstler darstellt. Gewonnen war mit der Idee des autopoietischen Bildes, für das die Photographie ein besonders prominentes Beispiel darstellen sollte, ${ }^{9}$ jedenfalls ein entscheidendes Argument für die Verpflichtung des Mediums auf die sichtbare Wirklichkeit, die sich unberührt von jeder künstlerischen Intention im photographischen Bild selbst zur Anschauung bringe.

Die alte Metapher des Silberspiegels gibt mit ihrer der Katoptrik verpflichteten Semantik einen deutlichen Hinweis auf die nicht geringen, an das photographische Bild gerichteten Erwartungen. Zwar ist den hinter dieser Metapher wirksamen repräsentationstheoretischen Stilisierungen vielfach und zu Recht widersprochen worden, dennoch scheinen sich auch die ästhetischen Prämissen des Photographischen in jene "Situation des `Gegenüber « ${ }^{10}$ einrücken zu lassen, von der Hans Jonas im Zusammenhang seiner Diskussion einer Phänomenologie der

6 William J. Mitchell: The Reconfigured Eye. Visual Truth in the Post-Photographic Era, Cambridge (Mass.), London 1992.

7 Andreas Müller-Pohle: Analogisieren, Digitalisieren, Projizieren. In: Silke Helmerdig, Martin Scholz (Hg.): Ein Pixel, Zwei Korn. Grundlagen analoger und digitaler Fotografien und ihre Gestaltung, Frankfurt am Main 2006, S. 98-109; hier S. 100.

8 Talbot 1844-1846 (wie Anm. 1), S. 89.

9 Und zwar seit der Pionierzeit des Photographischen. Siehe hierfür Kelley Wilder: William Henry Fox Talbot und "the Picture which makes ITSELF«. In: Friedrich Weltzien (Hg.): von selbst. Autopoietische Verfahren in der Ästhetik des 19. Jahrhunderts, Berlin 2006, S. 189197.

10 Hans Jonas: Der Adel des Sehens. Eine Untersuchung zur Phänomenologie der Sinne [1954]. In: Ralf Konersmann (Hg.): Kritik des Sehens, Leipzig 1997, S. 247-271; hier S. 268. 
Sinne sprach. "Das Sehen", so kennzeichnete Jonas den für die Wahrnehmung des photographischen Bildes gewiss wesentlichsten Sinn, "enthält in jedem gegebenen Augenblick eine unendliche Mannigfaltigkeit zugleich, und seine eigenen qualitativen Bedingungen öffnen den Weg in das, was jenseits liegt. Die Entfaltung des Raums vor dem Auge, unter dem Zauber des Lichts, trägt in sich den Keim der Unendlichkeit - als einen Aspekt des Sinnlichen selbst. " ${ }^{11}$ Dieser »Keim der Unendlichkeit « ist es, der als ein bedeutsames Potenzial nicht allein des Sehens im Allgemeinen ausgemacht werden kann, sondern auch des Sehens unter den spezifischen medialen Bedingungen des photographischen Bildes. Denn für die Wahrnehmung der Photographie ist nicht allein jenes wesentlich, was sich im Bild als Sichtbares konstituiert, sondern stets zugleich der hierin angelegte Verweis auf jenes, "was jenseits liegt«.

Die Bedeutung eines solchen Moments der Entgrenzung auf ein potenzielles 'Jenseits` hat Jonas mit Bezug auf eine Phänomenologie des Sehens näher gekennzeichnet: „Dies indefinite ,Undsoweiter`, mit dem die visuelle Wahrnehmung durchtränkt ist, ein stets bereites Potential für Aktualisierung, und besonders das 'Undsoweiter in die Tiefe des Raums, ist der Geburtsort der Idee von Unendlichkeit, für die kein anderer Sinn die empirische Basis liefern könnte. ${ }^{12}$ Im photographischen Bild ereignet sich dieses Zusammenspiel von Aktualität und Potenzialität unter medienästhetischen Bedingungen. Gewiss liegt einer der stärksten Gründe für die anhaltende Diskussion um die Wahrheitsfähigkeit des Photographischen in diesem engen Anschluss des Mediums an das von Jonas formulierte "indefinite ,Undsoweiter « visueller Wahrnehmung. Eine Identifikation von Sichtbarkeit im photographischen Bild und ihm vorgängiger Wirklichkeit - wie sie etwa in Barthes' Formel 'Ça a étér anklingt - wird jedoch stets zu kurz greifen müssen. Denn eine Photographie, die tatsächlich den "Keim der Unendlichkeit» in sich tragen soll, kann dies stets allein unter den Bedingungen ihrer spezifischen medialen Faktur leisten. Wahrnehmung von Unendlichkeit bleibt im photographischen Bild grundsätzlich an Fragen einer Poetik dieses Bildes gebunden. Anhand von drei verschiedenen künstlerischen Experimenten mit dem potenziellen photographischen Bild soll eine solche Poetik näher betrachtet werden.

II

Noch vor Zeichnung, Druckgraphik und Malerei, die allesamt bedeutenden Raum in David Hockneys künstlerischem Werk einnehmen, ist es die Photographie, die zum wesentlichen Gegenstand von Hockneys Auseinandersetzung um die Darstellungsmöglichkeiten des Bildes geworden ist. Die Rede vom >Bruchstück der Wirklichkeit gewinnt in diesen fortgesetzten Dekonstruktionen der visuellen

11 Ebd., S. 269-270. (Hervorhebung im Original.)

12 Ebd., S. 269. (Hervorhebung im Original.) 
Wahrnehmung und ihrer bildmedialen Repräsentation in reizvoller Weise ihren Sinn. Denn wenn in Hockneys photographischen Arbeiten der Akt des Sehens konsequent auf die Probe gestellt werden soll, so ist das wesentliche Mittel dieser Experimente eine sich bereits auf der Bildoberfläche überdeutlich abzeichnende Ästhetik der Fragmentierung. Der Riss zwischen Bild und Wirklichkeit wird bei Hockney in das Bild selbst hineinverlegt.

Anhand der großformatigen Photocollage "Pearblossom Highway« (Abb. 1) lassen sich solche Auseinandersetzungen mit der ästhetischen Grenze exemplarisch beobachten. ${ }^{13}$ Bereits einem flüchtigen Blick wird der brüchige Charakter der Bildoberfläche auffallen; erst recht aber begünstigt das große Format dieses Bildes eine genaue Untersuchung seiner Faktur. Hockneys Schilderung des kalifornischen Hinterlandes ist das Ergebnis einer Collage aus mehreren hundert Einzelphotographien, die zu einem umfassenden Tableau des Pearblossom Highway ausgelegt worden sind. Insbesondere im oberen Bilddrittel, das einen wolkenlosen Himmel zeigt, gibt sich dieses Verfahren visueller Addition ohne weiteres zu erkennen: Einem unregelmäßigen Schuppenkleid gleich sind hier Dutzende kleinformatige, beinahe monochrome Photographien an- und übereinander geschoben, so dass sich ein irisierender Wechsel zwischen Blau- und Weißtönen ergibt. Im unteren Teil des Bildes steht dieser nervösen Überlagerung einzelner Bildfelder eine in ihrem Effekt etwas glatter erscheinende Kombinatorik entgegen. Zwar ist auch hier die Ansicht von Steppe und Straßenbelag, Palmen und Straßenschildern brüchig, dennoch ist es mühelos möglich, die in der rechten Bildhälfte konzentrierten Schriftzüge allesamt zu lesen.

Hockneys Photocollage wahrt die klassischen Konventionen der Landschaftsdarstellung: Hierzu zählen die zentralperspektivische Einrichtung der Ansicht ${ }^{14}$ die hoch im Bild liegende Horizontlinie und ein weit in die Tiefe gestaffelter Bildraum, zu denen nicht zuletzt die Straßenschilder als Repoussoirfiguren beitragen. Und insbesondere ist der Riss zwischen Bild und Wirklichkeit, das heißt der äußere, diese Photocollage im Ganzen umschließende Rahmen konventioneller Natur: Das Tableau ist zu einem gleichmäßigen Längsrechteck abgeschnitten. Das Moment der Zersplitterung wird demgegenüber an den ungenauen Anschlüssen, an den Überlappungen und Aussparungen zwischen den einzelnen Photographien, das heißt an der Oberflächenstruktur der Collage kenntlich. Als würde der Bildraum implodieren, wird das Bild durch Widersprüche auf mikrostruktureller Ebene fragmentiert. Zudem lassen Differenzen in der Tonalität der Farben auf verschiedene Lichtverhältnisse schließen, die einer Entstehungszeit von beinahe

13 Mit Recht kann sie als Höhepunkt und Abschluss von Hockneys photographischen CollageExperimenten gelten. Siehe hierzu Anne Hoy: Hockneys Fotocollagen. In: Maurice Tuchman, Stephanie Barron (Hg.): David Hockney. Eine Retrospektive, Köln 1988, S. 54-65; hier insbesondere S. 55.

14 Zur Rolle der Perspektive in Hockneys Werk siehe Didier Ottinger: Éros perspectiviste. In: ders. (Hg.): David Hockney. Espace/Paysage, Paris 1999, S. 15-28. 


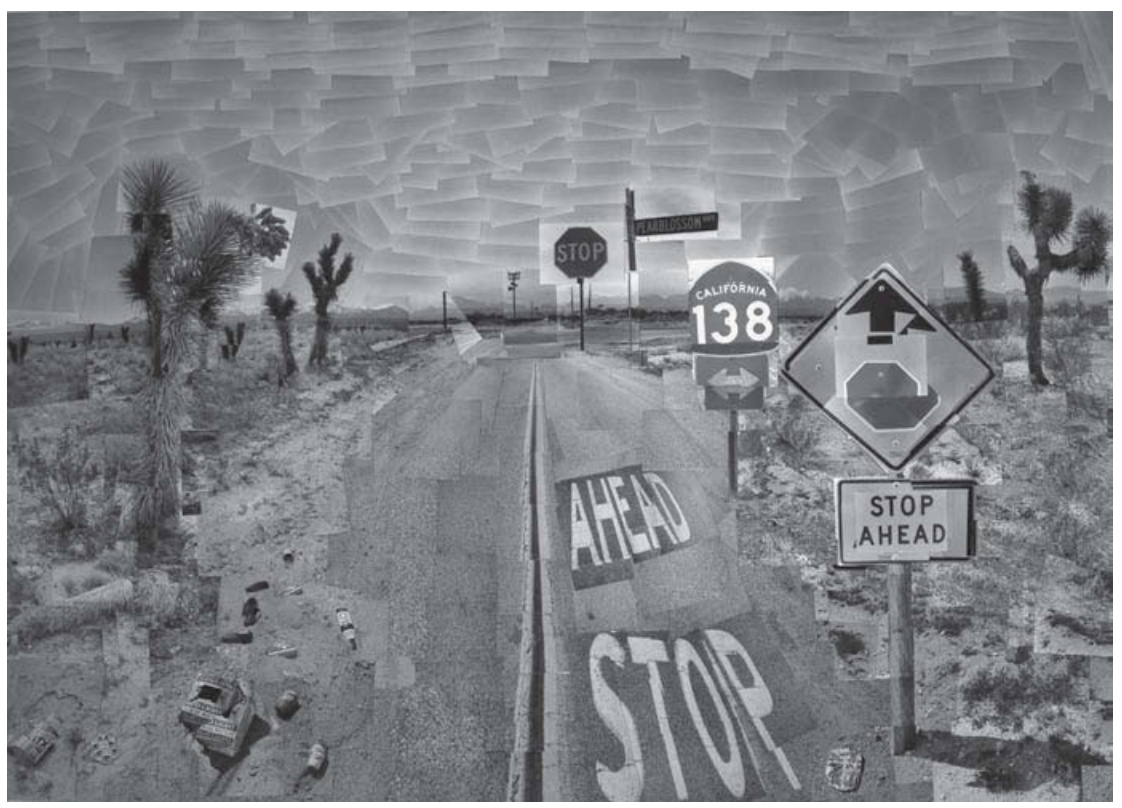

Abb. 1: David Hockney: Pearblossom Highway, 11-18th, April, 1986, \#1. Photocollage, 1986. $163,8 \times 119,4 \mathrm{~cm}$.

einer ganzen Woche geschuldet sind. So deutlich im Titel des Bildes Ort und Zeit markiert sind, so sehr verlieren sich in Hockneys Sammlung von Perspektiven und Augenblicken gerade diese Koordinaten eines klassischen Tafelbildes. Denn in der Tat geht es darum, waus zerbrochenen Repräsentationsstücken etwas Neues zu gewinnen. $\aleph^{15}$

In einer zweiten umfänglichen photographischen Werkgruppe löst Hockney die Stringenz dieser Koordinaten von Raum und Zeit vollends auf. Nicht mehr die Zusammenfügung vieler einzelner Bilder zu einem übergreifenden Bild, sondern vielmehr die Zergliederung eines solchen umfassenden Bildes in viele einzelne Bilder scheint die leitende Idee zu sein. Als überaus sinnfällig erweist sich eine solche analytische Operation insbesondere in der Collage "The Scrabble Game" (Abb. 2), hat es doch den Anschein, als solle hier mit photographischen Mitteln nachvollzogen werden, was auch Gegenstand des Spiels ist. Den einzelnen zum Kreuzworträtsel auszulegenden Buchstaben entsprechen die einzelnen kleinformatigen Bildfelder, denen sich erst durch sinnvolle Addition eine weiter reichende Semantik abgewinnen lässt. Darüber hinaus wird anhand der Mitspieler ein Moment der zeitlichen Dehnung kenntlich: Indem die Kopräsenz verschiedener Gesichts-

15 Merlin Carpenter: David Hockney, 1971. In: Texte zur Kunst Nr. 67 (September 2007), S. 270-279; hier S. 279. 


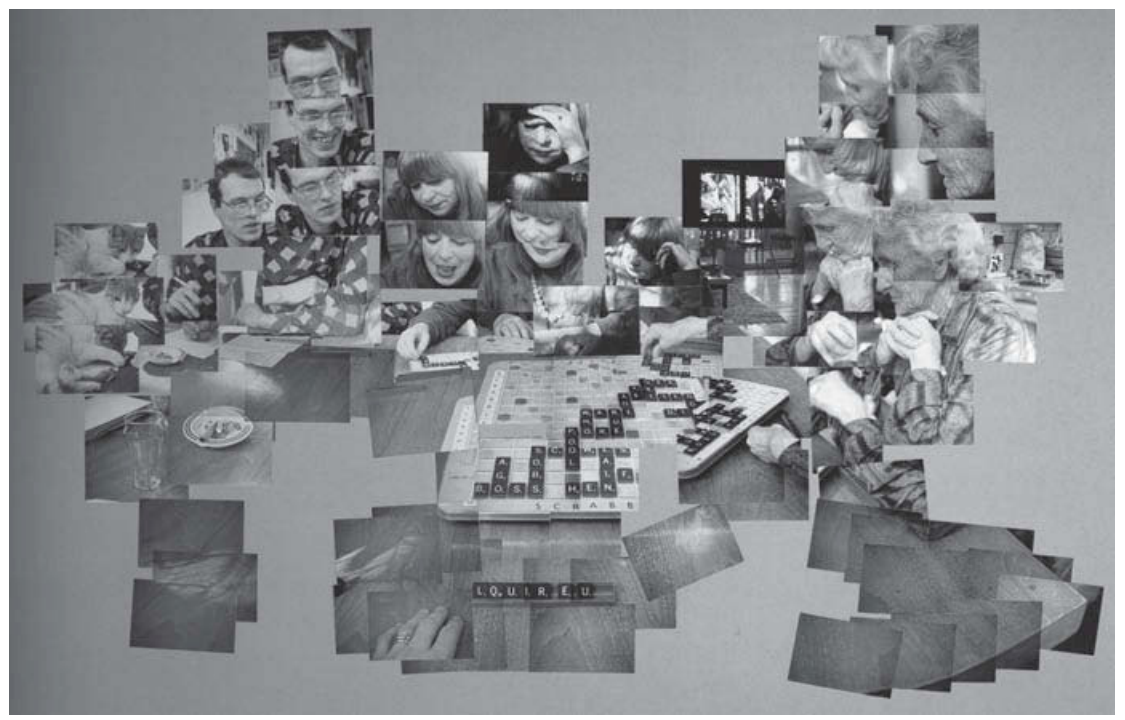

Abb. 2: David Hockney: The Scrabble Game, Jan. 1, 1983. Photocollage, 1983. Äußere Maße: $147,3 \times 99,1 \mathrm{~cm}$.

ausdrücke der jeweils selben Person auf verschiedene Phasen des Spiels verweist, wird die Collage als ein Zeit-Bild lesbar. ${ }^{16}$ Zum einen betrifft dies die im Bild präsentierte Handlung, zum anderen aber auch den Akt des Sehens und damit den Betrachter. Denn als könne sich die Sichtbarkeit eines Bildes überhaupt erst durch die Blickbewegungen des Rezipienten manifestieren, gibt die vollkommen unregelmäßige äußere Form dieses Bildes nur dort etwas zu erkennen, wo zuvor der Blick des Betrachters hinreichte. Ganz wesentlich bestehen diese Collagen daher nicht allein aus einer Vielzahl einzelner Photographien, sie gewinnen ihre Struktur zugleich durch den Einsatz von blinden, keiner näheren Bestimmung unterworfenen Feldern.

In Hockneys photographischen Collagen werden die rhetorischen Operationen von Pleonasmus und Ellipse kunstvoll miteinander verschränkt. Die sich in diesen Bilder ereignende Sichtbarkeit ist überreich und lückenhaft zugleich. Häufung und Verknappung sind ihnen gleichermaßen vorausgesetzt. Wenn damit jedes lineare Bildmaß gestaucht, gedehnt, gebrochen oder auch vervielfacht wird, so erinnert dies an die visuellen Strategien kubistischer Malerei und nicht zuletzt an

16 Siehe zu einer solchen Deutung auch Hoy 1988 (wie Anm. 13). Stefan Hesper: Kristalle der Zeit. Zur Anachronie der Wahrnehmung bei David Hockney und Gilles Deleuze. In: Jürgen Stöhr (Hg.): Ästhetische Erfahrung heute, Köln 1996, S. 126-147. Clive Scott: The Spoken Image. Photography and Language, London 1999, S. 219-228. 
die Versuche einer kubistischen Photographie. ${ }^{17}$ Wird der blinde Fleck als Bedingung des Sehens in Bildern üblicherweise nicht selbst thematisch, so ist er hier der wesentliche Gegenstand aller Darstellungsabsicht. Gerade indem diese Bildcollagen genauso viel verbergen wie sie zugleich zeigen, entfremden sie das photographische Bild nachdrücklich von der Idee einer mimetischen Reproduktion von Wirklichkeit. Und jene Idee des prägnanten Augenblicks, wie sie in der klassischen photographischen Ästhetik formuliert worden ist, wird in Hockneys kubistischen Tableaus in eine nicht mehr auszählbare Vielzahl von Perspektiven multipliziert. Sichtbarkeit im Bild wird auf diese Weise als ein Konstrukt kenntlich, das stets an latente Unsichtbarkeit grenzt. ${ }^{18}$ Wahrnehmung lässt sich daher nicht anders als ein Prozess fortgesetzter Neuorientierung im erkennbar unebenen Bildgelände auffassen. ${ }^{19}$

Folgerichtig verlieren sich in einer Vielzahl von Hockneys photographischen Collagen die sicheren Grenzen des rahmenden Rechtecks. Jedes in diesen Tableaus präsentierte Bildelement gibt sich dem Betrachter einzig in unregelmäßig geformten, scheinbar arbiträren Zonen von Sichtbarkeit zu erkennen. Die sich hierbei abzeichnende Idee von Transgression ist einem Effekt visueller Vorläufigkeit verpflichtet, gehört doch zu den Herausforderungen an den Betrachter, diese unmittelbar benachbarten Zonen der Unsichtbarkeit imaginativ in Sichtbarkeit zu übersetzen. Schließlich lässt sich eine solche nachdrücklich ästhetische Funktionalisierung des Rahmens in Hockneys photographischem Werk aber immer dann in genau entgegengesetzter Weise beobachten, wenn an Stelle des Kleinbildfilms Polaroidfilme zum Einsatz gelangen. Die für diese photographische Technik unverzichtbaren weißen Papierstege werden, wie etwa das Portrait "Gregory, Los Angeles, 31. März 1982« (Abb. 3) demonstriert, nicht beschnitten und damit also kaschiert, sondern als ein ubiquitäres Passepartout selbst zum Teil des Bildfeldes. Das Rechteck des Bildausschnitts verliert sich hier nicht in einem unregelmäßigen Äußeren, sondern verstärkt vielmehr ostentativ die quadratische Grundform des Polaroids. Zunächst gibt jede einzelne der sechzehn Aufnahmen einen nahsichtigen Ausschnitt des nach links gewendeten Kopfes zu erkennen. Zum übergreifenden Bildquadrat ausgelegt, wird diese Collage sodann aber in doppelter Weise lesbar: als ein kubistisches Bildnis und zugleich als ein regelmäßiges Raster weißer Rahmenstege. Und gerade dieses Raster ist es, das zwischen Werk und Beiwerk, zwischen Sichtbarkeit und Unsichtbarkeit changierend über die Prämissen von Hockneys photographischer Komposit-Ästhetik beredte Auskunft gibt.

17 Für ein Resümee dieses schon vielfach unternommenen Vergleichs siehe Christophe Blaser, Daniel Girardin: Der kubistische Raum in Hockneys Photocollagen. In: Reinhold Mißelbeck (Hg.): David Hockney. Retrospektive Photoworks, Heidelberg [1997], S. 33-40.

$18 \mathrm{Zu}$ diesem Zusammenhang von Darstellung und Undarstellbarkeit siehe Gottfried Boehm: Sehen. Hermeneutische Reflexionen. In: Internationale Zeitschrift für Philosophie 1 (1992), S. 50-67; insbesondere S. 61-67.

19 Siehe hierzu auch Hesper 1996 (wie Anm. 16). Alexandra Schumacher: David Hockney. Zitate als Bildstrategie, Berlin 2003, S. 97-115. 


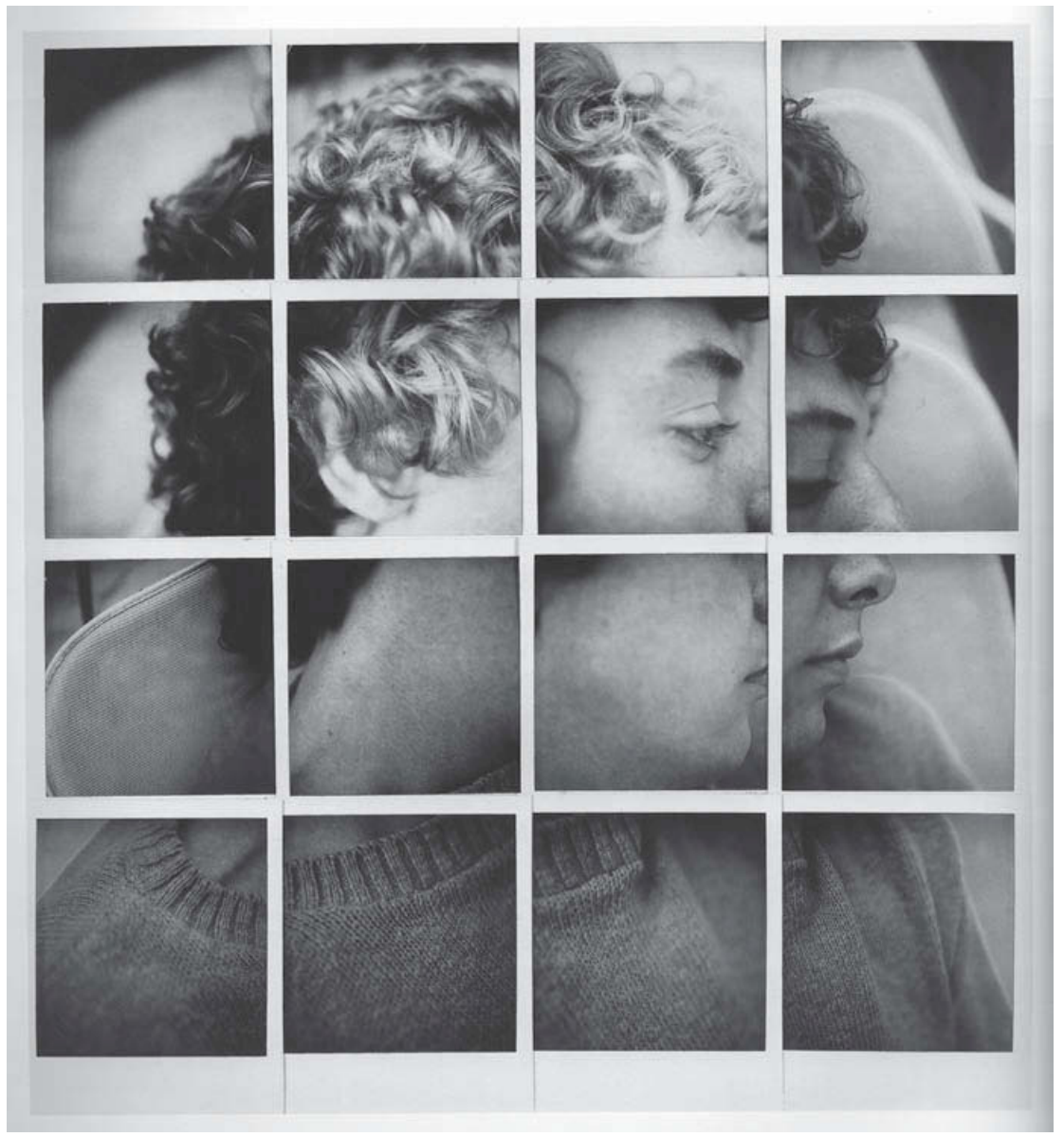

Abb. 3: David Hockney: Gregory, Los Angeles, 31. März 1982. Polaroid-Collage, 1982. 33,7 × $36,8 \mathrm{~cm}$.

\section{III}

Mit der Sichtbarkeit des Rahmens als Bild eines Rasters werden die apparativen Bedingungen des photographischen Mediums zum Gegenstand der Darstellung. Das selbstreflexive Moment, das sich hierin anzeigt, übersteigt jedoch gerade durch die Thematisierung des Rahmens als einem in das Bild hineinverlegten Element die klassische Ästhetik der Metaphotographie. ${ }^{20}$ Es ist die Latenz des Unsichtbaren,

20 Siehe hierzu Karlheinz Lüdeking: Vierzehn Beispiele fotografischer Selbstreflexion [2005]. In: ders.: Grenzen des Sichtbaren, München 2006, S. 19-38. 

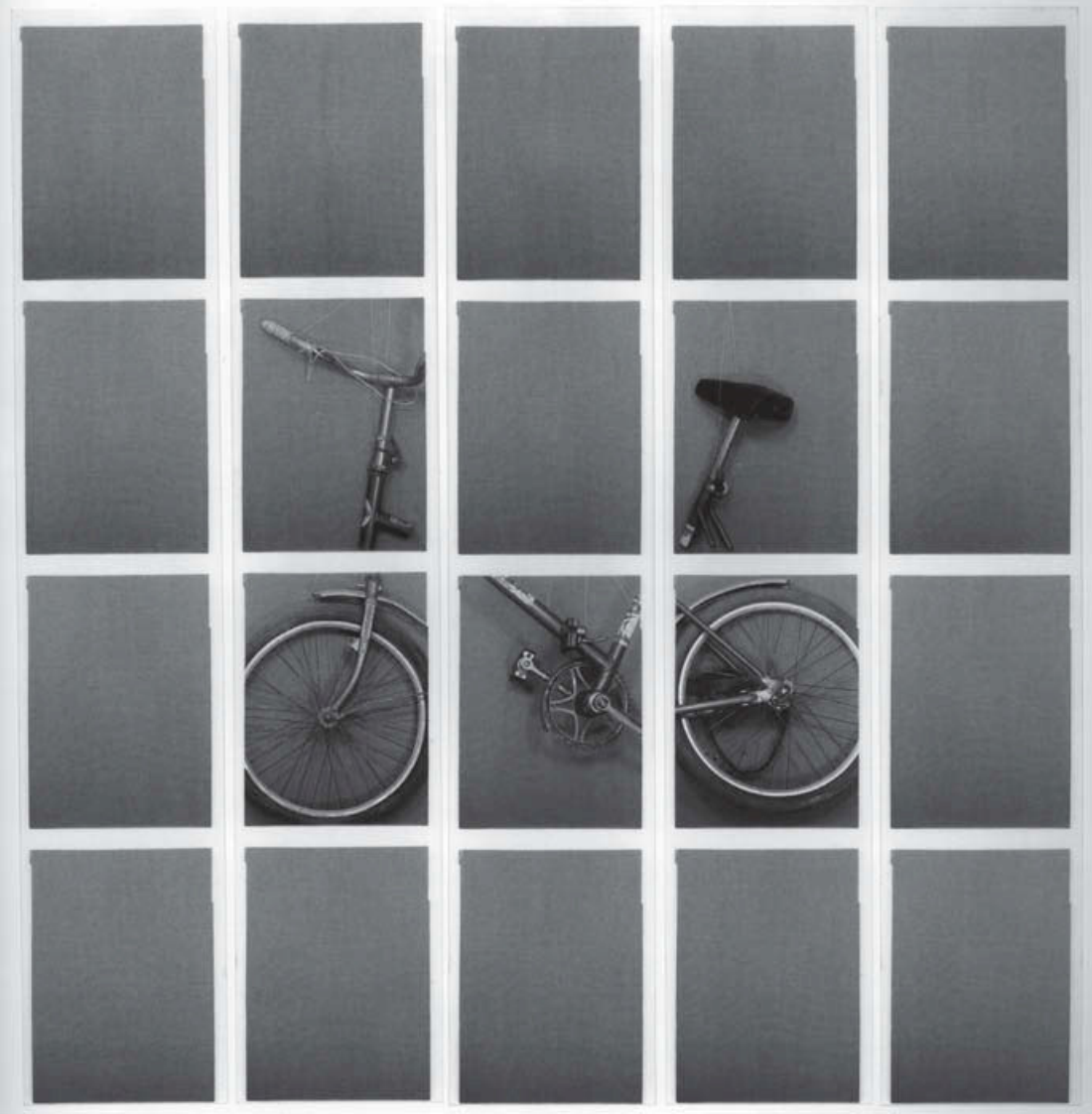

Abb. 4: Jan Wenzel: Fahrrad. Leipzig, Hauptbahnhof. Fotofix-Collage, 1996. 21,3 × 20 cm.

die sich in dieser Durchrasterung eines aus einzelnen Bildern collagierten Bildfeldes abzeichnet. Hockneys Vielfalt photographischer Verfahren macht es zuletzt jedoch unmöglich, diese mit einem umfassenden Begriff zu bezeichnen. Deutlich einfacher scheint dies demgegenüber möglich für die seit den frühen 1990er Jahren entstehenden Photo-Collagen des Leipzigers Künstlers Jan Wenzel. Eine Komposition aus zwanzig kleinen Bildfeldern wie in "Fahrrad. Leipzig, Hauptbahnhof" (Abb. 4) legt es nahe, von visueller Addition zu sprechen, die sich in ihrer Summe zu einem Bild mit einer überaus klaren, streng orthogonalen Struktur fügt. Bei genauerem Hinsehen wird sich jedoch erweisen, dass es sich bei Wenzels Bildern stets um Additionen mit außerordentlich ungleichen Größen handelt, zu denen nicht selten überdies visuelle Unbekannte treten, die zu entschlüsseln keine geringe, an den Betrachter gerichtete Aufgabe ist. 
Nur fünf der insgesamt zwanzig hochrechteckigen Bildfelder genügen Wenzel, um das Fahrrad abzubilden; die übrigen fünfzehn Felder hingegen geben nicht mehr als einen gleich bleibenden, warmen Hintergrundton zu erkennen, der zwischen Karminrot und Orange changiert. Wenzel selbst hat darauf hingewiesen, dass sich die von ihm photographierten Objekte und Kulissen als objets trouvés des mit den Jahren 1989/1990 untergegangenen DDR-Alltags auffassen lassen. ${ }^{21}$ Und dementsprechend handelt es sich bei diesem Fahrrad um das sichtlich desolate Modell eines MIFA-Klapprades. Bei näherer Betrachtung der Konstruktion wird jedoch deutlich, dass dieses Fahrrad nicht allein ramponiert, sondern längst vollkommen unbrauchbar geworden ist. Lenker und Sattel wurden ganz offenbar mit sauberen Schnitten vom Rahmen getrennt, und es besteht aller Grund zur Annahme, dass auch dort, wo dies nicht anhand der einzelnen Bildfelder sichtbar wird, nur noch Einzelteile auf ein ehemals intaktes Fahrrad verweisen. Zuallererst handelt es sich bei dieser Komposition aus zwanzig Bildfeldern nicht um das übergreifende Bild eines Fahrrads vor einem orange-roten Dekor, sondern um isolierte Einzelbilder monochromer Farbflächen sowie separierter Teile eines Fahrrads.

Wenzels Kompositionen sind das Ergebnis eines präzise geplanten und außerordentlich aufwändig eingerichteten visuellen Spiels mit den technischen Möglichkeiten des Fotofix-Apparates, wie er sich etwa in Bahnhöfen, Behörden oder Fußgängerpassagen finden lässt. ${ }^{22}$ Und es ist die prinzipiell auf die Zahl 4 zählende Syntax dieser Sofortbilder, die sich in Wenzels Photographien nachdrücklich niederschlägt: Denn stets werden diese Bilder aus vertikal ausgerichteten Streifen von je vier Fotofix-Bildern zusammengesetzt, so dass in der horizontalen Ordnung zwischen den einzelnen Streifen zwar schmale Lücken stehen bleiben, in der vertikalen Ordnung hingegen eine lückenlose und unbeschnittene Folge von Bildfeldern sichtbar wird. Ein solcher Verweis auf die Lückenlosigkeit ist wichtig, da gerade hierdurch diese Bilder eine ganz wesentlich zeitliche Dimension erhalten: Im Unterschied zu David Hockneys einzelnen Kleinbildphotographien und Polaroids, die stets erst nachträglich zu großen Landschaftstableaus oder auch intimen Bildnissen ausgelegt worden sind, folgen Wenzels vertikale Sequenzen den apparativen Bedingungen des Fotofix-Automaten, der in einem nur nach Sekunden getakteten Rhythmus eine Gruppe von je vier Bildern produziert. Die sich in den

21 Jan Wenzel: From the Garbage into the Booth - oder: Sofortbilder eines umgestülpten Alltags. In: Klaus Kleinschmidt (Hg.): Fotofix. Jan Wenzel, Heidelberg 2005, S. 8-11. Wieder abgedruckt in Meike Kröncke, Barbara Lauterbach, Rolf F. Nohr (Hg.): Polaroid als Geste über die Gebrauchsweisen einer fotografischen Praxis, Ostfildern-Ruit 2005, S. 74-79.

22 Zu Prinzip und Geschichte dieses photographischen Dispositivs siehe Rolf Behme: Foto-Fix. Es blitzt viermal, Dortmund 1996, insbesondere S. 7-15. Rolf Nohr: A Dime - A Minute A Picture. Polaroid \& Fotofix. In: Petra Löffler, Leander Scholz (Hg.): Das Gesicht ist eine starke Organisation, Köln 2004, S. 160-180. Roland Meyer: Aufnahmebedingungen. Versuchsanordnung Fotoautomat. In: Inge Hinterwaldner et al. (Hg.): Topologien der Bilder, München 2008, S. 99-116. 


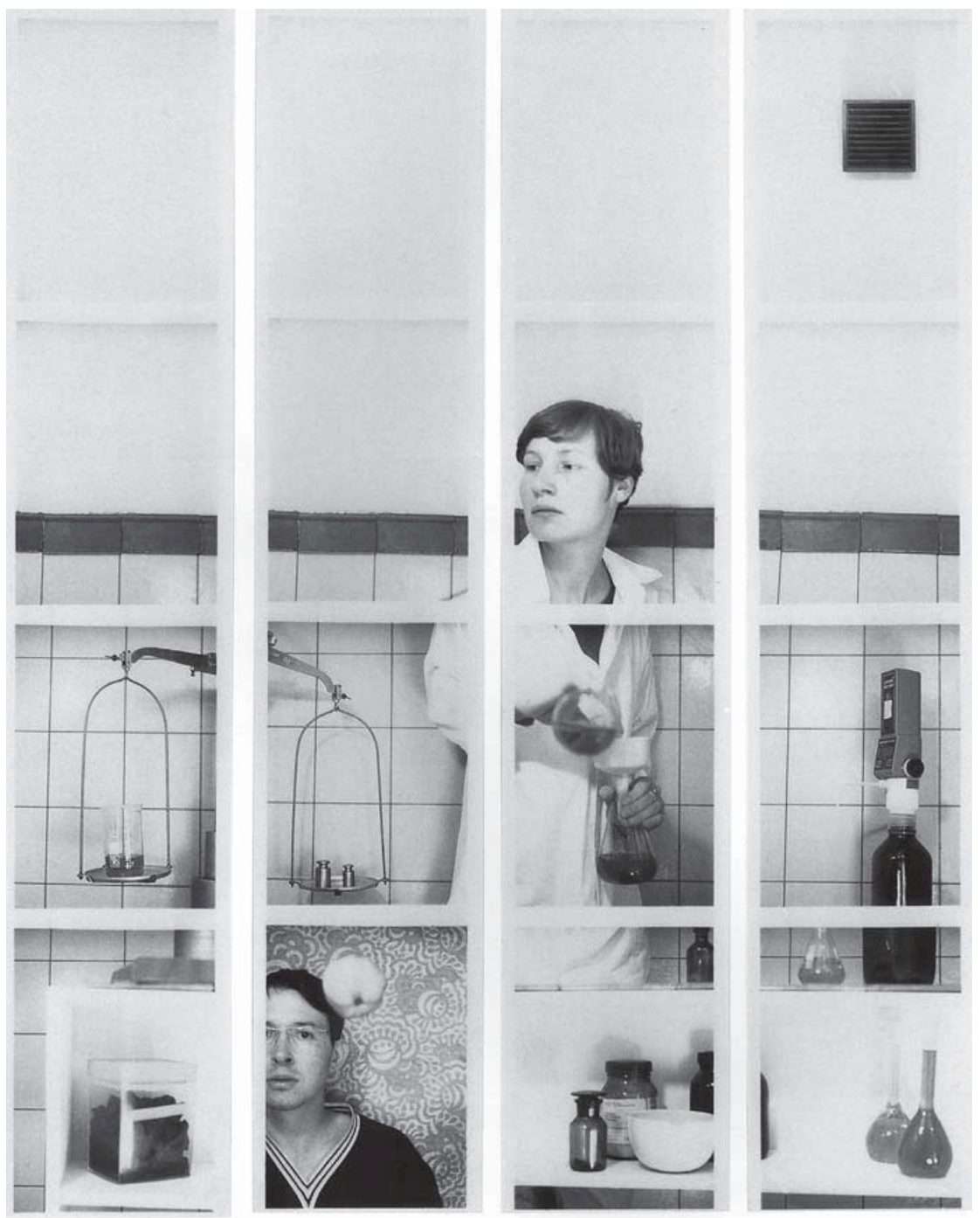

Abb. 5: Jan Wenzel: Interieur XIII. Leipzig, Prager Straße. Fotofix-Collage, 1998. 16× 21,3 cm. 
vertikalen Achsen abzeichnenden Differenzen müssen daher als das Ergebnis einer außerordentlich raschen (oder eben "fixen«), der unerbittlichen Folge von Belichtungen gehorchenden Verwandung des photographischen Objekts interpretiert werden. ${ }^{23}$

Wie kunstvoll sich Wenzels experimenteller Ludismus steigern lässt, wird anhand des Bildes "Interieur XIII" (Abb. 5) augenfällig: Zur Darstellung scheint hier - wie die hellen Fliesen, die farbigen Kolben, die Waage und andere Apparaturen anzeigen - ein chemisches Labor zu gelangen. Und insbesondere ist in den mittleren Feldern dieser Bilder-Komposition ein Chemiker zu erkennen, der gerade eine rote Flüssigkeit in einen Erlenmeyerkolben füllt. Jedes dieser einzelnen, ihrem eigentlichen Zweck nach nur passbildgroßen Felder ist hier Gegenstand einer intensiven Ausstattung mit Kulissen und Requisiten. Der Kasten des Automaten wird auf diese Weise von Wenzel zu einer Bühne umgedeutet, auf der sich im Halbminutentakt und in vier Akten ${ }^{24}$ je neue Szenen aufführen lassen. Sind diese einzelnen photographischen Szenen für sich genommen nur wenig oder zumeist überhaupt nicht aussagekräftig, so laden sich diese visuellen Einzelheiten in ihrer Gesamtheit zu einem kohärenten Ganzen auf. Gerade dieses Zusammenspiel führt zu einer Summe, die sich mit größerem Gewinn deuten lässt. Umso augenfälliger nimmt sich daher in diesen additiven Tableaus die Ausnahme von der Regel aus: Für den Fall des "Interieur XIII" handelt es sich um das zweite Bild in der unteren Zeile, das vollkommen unvermittelt in die semantische Logik dieses Labors einbricht und einem Fremdkörper gleich wie aus einer anderen Bildordnung aufzutauchen scheint.

Anschlüsse sind in Jan Wenzels photographischen Kompositionen die wesentlichen Agenten der Bildlogik. In den Vertikalen folgen sie einer durch die Apparatur des Fotofix-Automaten definierten iterativen Ordnung. Es ist diese kaum mehr als zwei Minuten umfassende zeitliche Abfolge, die von Wenzel in eine räumliche Iteration übersetzt wird. Die horizontale Orientierung indes kann, was in den Bildern kunstvoll überspielt wird, weit größere Abstände umspannen. Soll die räumliche Logik der inszenierten Szenen gewahrt bleiben, so muss der Ausführung im Automaten eine äußerst präzise Reflexion über die später zu addierenden Elemente vorausgehen. Wenn anhand dieser Folgen von Sofort-Bildern eine Miniaturwelt zusammengesetzt werden soll, so ist ihr das Konzept eines gesamten Tableaus vorausgesetzt, das zunächst einzig imaginär existiert. Wenzels Bild-Kompositionen sind daher stets das Ergebnis einer dreifachen Fertigkeit: genaue Planung der Szenen, handwerkliche Perfektion bei der Anfertigung der Kulissen und

23 Siehe hierzu auch Klaus Kleinschmidt: Jan Wenzel - Fotofix. In: ders. (Hg.): Fotofix. Jan Wenzel, Heidelberg 2005, S. 4-5. Zu älteren künstlerischen Auseinandersetzungen mit dem Dispositiv der Automatenphotographie siehe Behme 1996 (wie Anm. 22), insbesondere S. 17-30.

24 Einen solchen Vergleich zog bereits Klaus Kleinschmidt. Siehe Kleinschmidt 2005 (wie Anm. 23), S.5. 

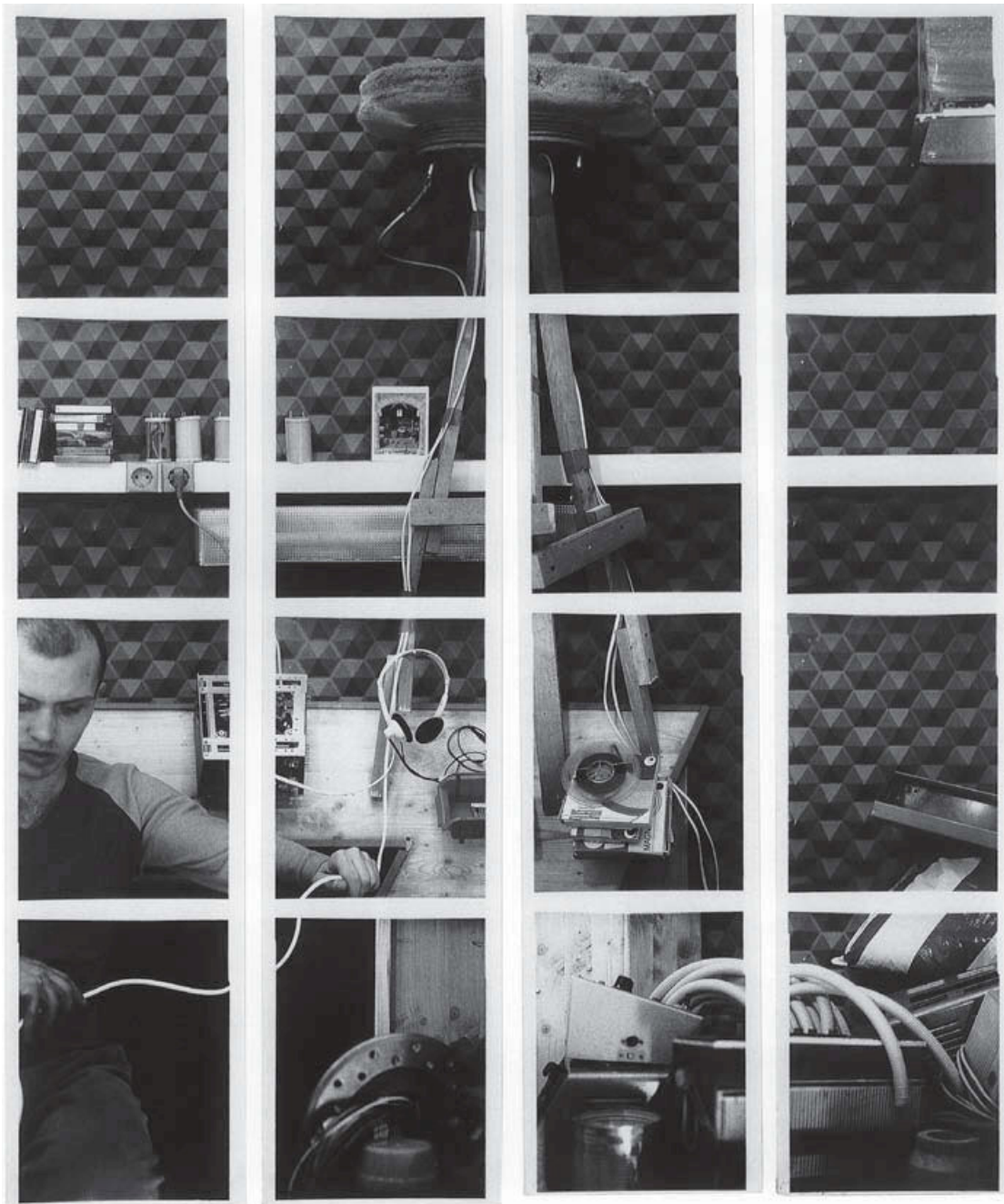

Abb. 6: Jan Wenzel: Bastler V. Leipzig, Prager Straße. Fotofix-Collage, 1999. $16 \times 21,3 \mathrm{~cm}$. 
Requisiten und präzise Aufführung vor der Kameralinse. Alles andere als ein Zufall scheint es daher zu sein, dass eine der Serien einen Bastler in seiner Werkstatt zum Gegenstand hat.

Wenn Wenzel selbst in dieser Selbstthematisierung eine Anspielung auf Claude Lévi-Strauss' Konzept der `bricolage sieht ${ }^{25}$ so verbindet sich mit dieser der Produktionsästhetik geltenden Bezugnahme in »Bastler $V_{«}$ (Abb. 6) zugleich ein unauffälliger Hinweis auf das bildmediale Prinzip dieser Kompositionen: Das zweite Bild in der zweiten Reihe zeigt, auf einem Regalbrett an die Wand gelehnt, eine Reproduktion von Antonello da Messinas "Hieronymus im Gehäuse«. Antonellos Gemälde ist eine Meditation über das Verhältnis von innen und außen; in der kunstvollen Verschränkung mehrerer Interieurs, die in einer sukzessiven Verschachtelung einander umschließen, wird der Raum, in dem Hieronymus sitzt, als eine iterative Staffel von Räumen sichtbar. In ihrem Kern gibt sie dem Kirchenvater, der über seiner Bibelübersetzung sitzt, Schutz gegenüber der Außenwelt. Bei Wenzels Bild einer Werkstatt hingegen wird dieses Prinzip der Einhüllung gegen sich selbst gekehrt: So logisch sich die Anschlüsse von Bildfeld zu Bildfeld ausnehmen mögen, faktisch steht keines der Bilder mit seinem nächsten in Verbindung. Ihre übergreifende Ordnung bleibt eine imaginäre Konstruktion. Als ein Trompe l'œil ist sie dem Auge des Betrachters zugänglich. Den Blick auf das Raster von weißen Randstegen und Lücken zu richten, wird indes heißen, der Abgründigkeit dieser trügerischen Bildsummen gewahr zu werden. ${ }^{26}$

IV

Den apparativen Bedingungen des Fotofix-Automaten geschuldet, ereignen sich Jan Wenzels Akte einer methodischen Zergliederung sowie Zusammenfügung der photographischen Bildfläche im Passbildformat. Von gänzlich anderem Maß nimmt sich demgegenüber das durch den Hamburger Künstler Peter Hendricks im Jahr 2003 angestoßene Ravensburgprojekt aus. ${ }^{27}$ Denn beinahe stets zu Gruppen von drei oder auch mehr großformatigen Tafeln kombiniert, fügen sich Hendricks' Photographien zu raumgreifenden Arbeiten von mitunter mehr als vier Metern Breite. Mit dieser eindrucksvollen physischen Präsenz geht zudem, wie ein Blick auf "Marienplatz im Mai 2003, I.1-I.5« (Abb. 7) zeigt, eine inhaltliche Fülle dieser Bilder einher, die ganz darauf angelegt zu sein scheint, einen bloß flüchtigen Blick auf diese Tableaus schnell zu überfordern. Als wolle Hendricks mit

25 Wenzel 2005 (wie Anm. 21), S. 11 beziehungsweise S. 79.

26 Für den Versuch einer literarischen Adaption dieses kompositorischen Prinzips siehe Friederike Mayröcker: vom Winterschreiben [1998]. In: dies.: Magische Blätter V, Frankfurt am Main 1999, S. 10-12.

27 Peter Hendricks: Das Ravensburgprojekt, hg. von Claudio Hils und Thomas Knubben, Köln 2004. 

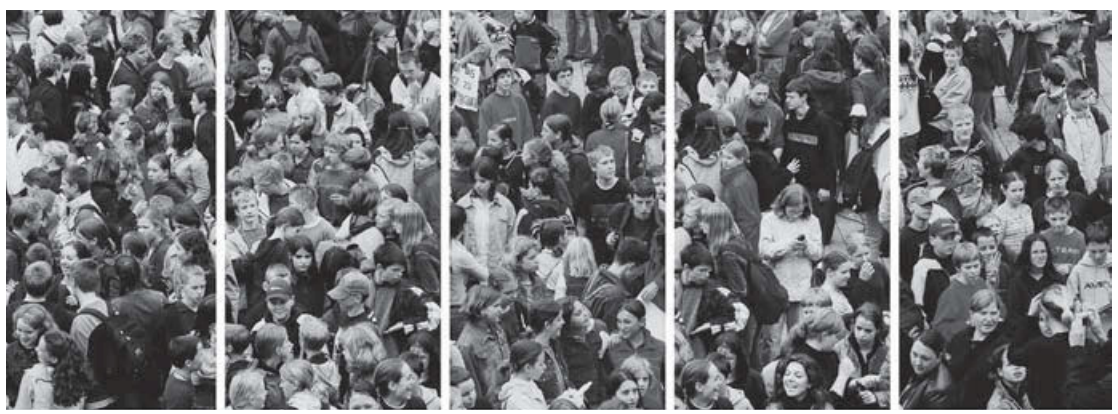

Abb. 7: Peter Hendricks: Das Ravensburgprojekt: Marienplatz im Mai 2003, I.1-I.5. LambdaPrints, 5 Tafeln, insgesamt $440 \times 166 \mathrm{~cm}$. Siehe auch Farbtafel XVI.

dem Bild der Menge ein traditionsreiches Motiv der bildenden Künste wieder aufgreifen, ${ }^{28}$ wimmelt auf den insgesamt fünf hochformatigen Tafeln eine nur schwerlich bestimmbare Zahl von Schülern, die, wie der Titel zu erkennen gibt, sich im Mai des Jahres 2003 auf dem Marienplatz in Ravensburg aufgehalten haben müssen.

Der durch den Photographen gewählte Standpunkt ist eine doppelbödige Einladung an den Betrachter. Denn aus einer deutlich erhöhten Position photographiert, kann sich der hier inszenierte Blick auf den Marienplatz und insbesondere auf seine Besucher vor jedem Zurückblicken sicher fühlen. Photograph und Betrachter teilen die Perspektive eines Voyeurs, der, wohl von einem Turm schauend, sieht, ohne selbst gesehen zu werden. Scheint dieses Moment der ’Okulartyrannis ${ }^{29}$ ohnehin ein Signum der photographischen Praxis zu sein, so wird es bei Hendricks zum ästhetischen Prinzip gewendet. Denn es ist erkennbar, dass in dieser photographischen Beschreibung einer »demoskopischen Landschaft « ${ }^{30}$ gerade der allmächtige, sich entrückt gebende Blick auf eine arglose und gerade dadurch sich spontan verhaltende Menschenmenge im Mittelpunkt des künstlerischen Interesses steht. Ganz im Sinn der Street Photography ist Hendricks Ravensburgprojekt eine soziologische Feldforschung, die sich gegenüber ihrem Untersuchungsobjekt deutlich auf Abstand hält. ${ }^{31}$ Zum fünffachen Tableau ausgebreitet, wird es Photograph wie Betrachter möglich, Verhalten und Habitus jener (pubertierenden) Jugendlichen eingehend zu studieren, die im Bewusstsein, beobachtet zu werden, vermutlich kaum ihre interesselose Teilnahmslosigkeit gewahrt hätten.

$28 \mathrm{Zu}$ den vielfältigen Voraussetzungen eines solchen Bildtyps siehe insbesondere Wolfgang Kemp: Das Bild der Menge (1789-1830). In: Städel-Jahrbuch N.F. 4 (1973), S. 249-270.

29 Astrit Schmidt-Burkhardt: Okulartyrannis. Vom Foto-Auge zum Kamera-Auge. In: Fotogeschichte Heft 100 (2006), S. 37-49.

30 Ulf Erdmann Ziegler: Conditio humana Ravensburg. In: Peter Hendricks: Das Ravensburgprojekt, hg. von Claudio Hils und Thomas Knubben, Köln 2004, S. 10-19; hier S. 10.

31 Ute Eskildsen (Hg.): Street \& Studio. An Urban History of Photography, London 2008. 
Hat man einmal einen solchen studierenden, insbesondere den Details geltenden Blick gegenüber diesen Bildern eingenommen, so wird jedoch schnell ersichtlich, dass die hier inszenierte Beobachtungssituation unzuverlässig ist. Das von Hendricks gesammelte und in Großformaten präsentierte Datenmaterial ist redundant und lückenhaft zugleich: Ein blondes Mädchen etwa, das einen leuchtend roten Anorak trägt, ist in identischer Haltung am rechten Rand der zweiten Tafel und in der linken Bildhälfte der vierten Tafel zu sehen; auf der mittleren Tafel schließlich ist sie im oberen rechten Viertel ein drittes Mal, nun jedoch vom Betrachter abgewendet, sichtbar. Angesichts dieser Wiederholungen und Variationen drängt sich die Frage auf, ob diese Menge von Jugendlichen tatsächlich so groß gewesen ist, wie das mächtige, aus fünf Bildern gefügte Tableau nahe legen will. Ganz scheint es jedenfalls so, als seien die schmalen Lücken zwischen den einzelnen Bildtafeln, durch die die Komposition rhythmisiert wird, als Unterbrechungen eines übergreifenden Kontinuums grundsätzlich nicht angemessen gedeutet; selbst wenn, wie dies zwischen der ersten und der zweiten Tafel (und dort am unteren Ende vor allem anhand des Schnitts durch den Kopf eines Jungen) zu beobachten ist, eine solche einfache Trennung mitunter dennoch der Fall sein kann.

Innen und Außen, Sichtbarkeit und Unsichtbarkeit stehen in Hendricks photographischen Kompositionen in einem prekären Verhältnis zu einander. Die Verwirrung, die sich bei einer näheren Beobachtung dieser großformatigen Felder einstellen wird, ist jedoch nicht allein Teil des künstlerischen Spiels mit Rezeptionserwartungen; es greift vielmehr auch auf die Produktion dieser Bildfolgen aus. Das indexikalische Moment, das diese Bilder in ihrem Gestus scheinbar objektiver, in jedem Fall aber unbeteiligter Beobachtung evozieren, ist längst selbst Gegenstand der Beobachtung geworden. Der von Hendricks einmal gesammelten, photographisch sicher gestellten Datenmenge lässt sich nur dann gerecht werden, wenn sie in immer neuen Variationen einer visuellen Untersuchung unterzogen wird. Ein Beleg hierfür ist etwa die Bildfolge "Marienplatz im Mai 2003, III.1III.5 " (Abb. 8), die deutlich nahsichtiger und daher einzig in Ausschnitten einige wenige Jugendliche präsentiert. Einmal mit Hendricks ästhetischem Prinzip der Zergliederung vertraut, wird auch hier die Suche nach richtigen und falschen Anschlüssen, nach Lücken und Wiederholungen rasch einsetzen. Und es handelt sich, wie ein Vergleich mit "Marienplatz im Mai 2003, I.1-I.5 « erweist, wenigstens in Teilen um Ausschnitte aus der rechten oberen Ecke im ersten Bildfeld dieses größeren, fernsichtigen Tableaus. Die ins Profil gewendeten Köpfe zweier Jugendlicher ließen sich bereits dort inmitten der Menge mühelos ausmachen.

So wie sich der Betrachter auf diese Weise mit immer neuen Beobachtungsversuchen diesen Tafeln nähern kann, so scheint auch der Photograph selbst ein Beobachter seiner eigenen Bilder, deutlicher noch: ein "Voyeur am eigenen Material $\aleph^{32}$ gewesen zu sein. Es ist das in Antonionis Film präludierte Verfahren des

32 Ziegler 2004 (wie Anm. 30), S. 13. 

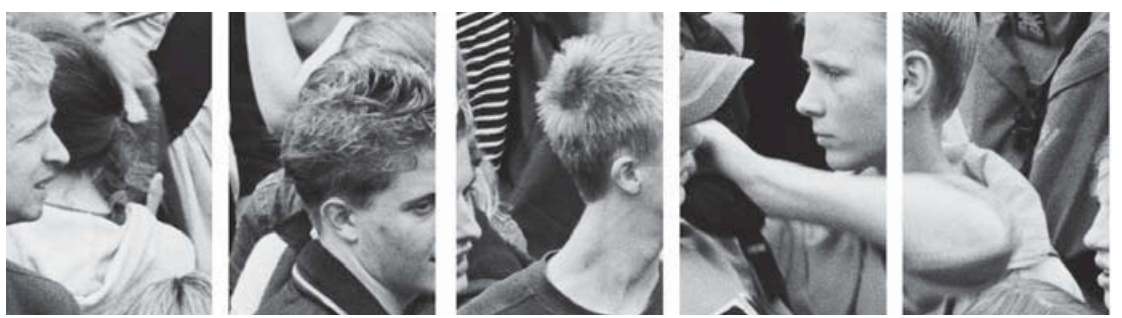

Abb. 8: Peter Hendricks: Das Ravensburgprojekt: Marienplatz im Mai 2003, III.1-III.5. LambdaPrints, 5 Tafeln.

'blow up ‘, also der immer weiter in das Bildmaterial dringenden Ausvergrößerung einzelner Szenen, das Hendricks Ravensburgprojekt in seiner Gesamtheit überspannt. Sichtbar wird hierbei jedoch nicht, wie dies noch in Antonionis Film aus dem Jahr 1966 der Fall war, die Körnung des Analogfilms; zur Erscheinung kommen vielmehr die diskreten Pixel des digitalen Bildes. ${ }^{33}$ Doch steht das dubitative, sich mikrostrukturell manifestierende Moment, ${ }^{34}$ das sich mit einer solchen Technik sukzessiver Immersion in das photographische Material verbindet, bei Hendricks gegenüber einem makrostrukturellen Interesse an einem Dementi der Erwartung von Referenzialität deutlich zurück. Es ist, wie die Bildfolge "Marienplatz im Mai 2003, III.1-III.5 « vor Augen stellt, das Zusammenspiel sich erfüllender und sich entziehender Bildanschlüsse, die hier mit größerem Nachdruck den Betrachter an der visuellen Logik dieser photographischen Kombinatoriken zweifeln lassen.

Das zweite und das dritte Bildfeld etwa oder auch das vierte und das fünfte Bildfeld sind, insbesondere durch die jeweils rücksichtslos zerschnittenen Köpfe, problemlos miteinander verknüpfbar. Auch zwischen dem zweiten und dem dritten Bildfeld stellt sich ein solcher Anschluss flüchtig ein, obwohl rasch deutlich wird, dass Hinterkopf und Schirmkappe zwei vollkommen unterschiedlichen Personen angehören. Lässt man sich auf diese Suche weiter ein, so kann man schließlich enträtseln, dass der linke Rand des ersten Bildfeldes an den rechten Rand des dritten Bildfeldes anschließen müsste, die kombinatorische Bildsyntax also auf einem Tausch beruht. Ein solcher Eingriff betrifft nicht allein die Logik der einzelnen Bildfelder sowie die unter diesen gestifteten Beziehungen. Gerade die Zwischenräume, das heißt die Lücken zwischen den Bildern sind es vielmehr, die sich hier als wesentliche Agenten visueller Transgression interpretieren lassen. Mitunter kann jenes unsichtbare Jenseits, von dem Hans Jonas schrieb, anhand von Hen-

33 An dieser Stelle sollte nicht unerwähnt bleiben, dass die Arbeiten von Peter Hendricks zunächst auf analogem Bildmaterial basieren, das erst anschließend digital weiterverarbeitet wird.

34 Siehe zu einer solchen Deutung digitaler Photographie Peter Lunenfeld: Digitale Fotografie. Das dubitative Bild [2000]. In: Herta Wolf (Hg.): Paradigma Fotografie. Fotokritik am Ende des fotografischen Zeitalters, Frankfurt am Main 2002, S. 158-177. 

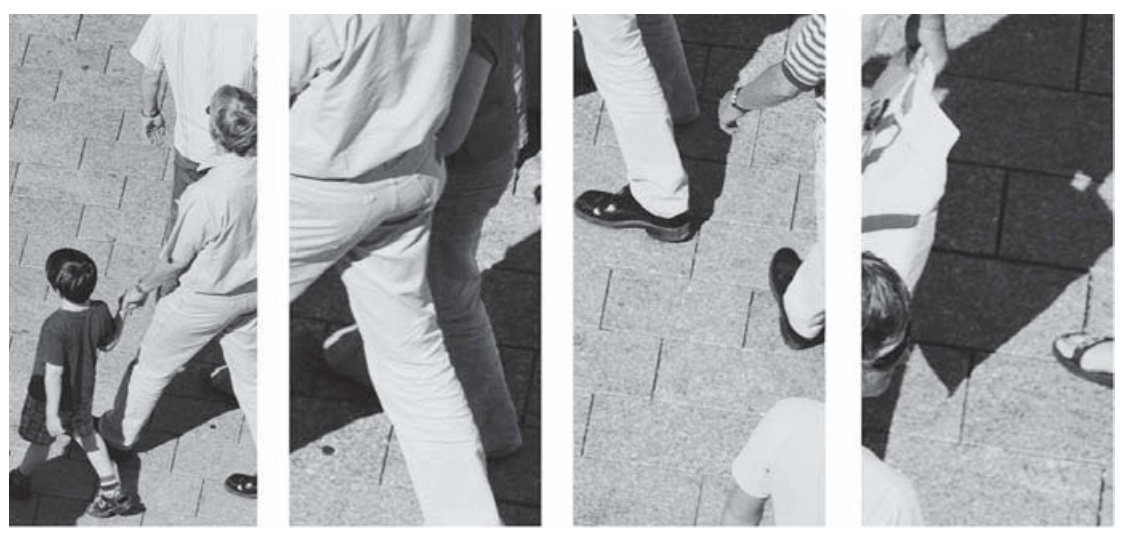

Abb. 9: Peter Hendricks: Das Ravensburgprojekt: Marienplatz im September 2003, IV.1-IV.4. Lambda-Prints, 4 Tafeln, insgesamt $155 \times 80 \mathrm{~cm}$.

dricks' Bild-Kombinatoriken in Sichtbarkeit übersetzt werden, mitunter scheint es sich aufgrund eines solchen umfassenden Spiels von Einsetzungen wie von selbst abzuzeichnen. Vollends gesteigert wird ein solcher Effekt potenzieller visueller Anschlüsse schließlich in einer Bildfolge wie »Marienplatz im September 2003, IV.1-IV.4« (Abb. 9), die mit verschiedenen Stufen der Vergrößerung und damit der Nahsichtigkeit arbeitet, um die - Bild für Bild genommen - kaum noch verständlichen Einzelmotive in einer Komposition zusammenzurücken, die in sich äußerst brüchig bleibt. Umso nachdrücklicher jedoch werden gerade hierdurch die Valenzen einer zwischen Sichtbarkeit und Unsichtbarkeit operierenden potenziellen Photographie erkennbar.

\section{V}

Ausgangspunkt jeder photographischen Ästhetik, so schrieb Rosalind Krauss, ist »die Anerkennung des Ausschnitts, des Abschneidens, die Tatsache, daß Photographie, wenn sie die Welt verdoppelt, dies nur stückweise tut. $\AA^{35}$ Wenn dieser von Krauss angesprochene, für jedes einzelne photographische Bild behauptete Charakter des Fragmentarischen in Frage steht, so sind es insbesondere die Techniken der Abschneidens und der Abgrenzung, sodann aber auch des Anschlusses und der Überschreitung, die für eine Diskussion potenzieller Bildlichkeit von besonderem Interesse sind. Denn solche Potenziale entfalten sich nicht allein innerhalb

35 Rosalind Krauss: Stieglitz Äquivalente. In: dies.: Das Photographische. Eine Theorie der Abstände, [Paris 1990], übers. von Henning Schmidgen, München 1998, S. 127-137; hier S. 131. 
einer durch Rahmung in ihrer Ausdehnung definierten Bildfläche; ${ }^{36}$ sie können vielmehr, dies kann der Blick auf die Arbeiten von Hockney, Wenzel und Hendricks erweisen, gerade auch jenseits dieser von außen an das Bild getragenen Grenzsetzungen siedeln. Der von Hans Jonas angesprochene "Keim der Unendlichkeit« berührt hierbei nicht allein die Grundlagen des Verstehens, sondern auch des Redens über Bilder. Mit der seit Leon Battista Alberti geläufigen Fenster-Metapher ist eine Vorstellung von Bildlichkeit wirksam, die den Charakter des Fragmentarischen durch die aufeinander bezogenen Operationen von Ausgrenzung und Synthese zu modellieren versucht. ${ }^{37}$ Das,$I n n e n `$ des Bildes steht in einer solchen Konzeptualisierung nicht allein gegenüber einem abgetrennten `Außen`; die Rede von einem solchen 'Innen`stellt vielmehr die Voraussetzung einer autonomen, das heißt einzig einer Logik des Bildes verpflichteten visuellen Ordnung dar. Jedes Moment faktischer oder auch nur prätendierter Transgression dieser Rahmenfunktionen stellt daher den Ausgangspunkt zur Diskussion um die Optionen bildlicher Ästhetik oder emphatischer und mit den Worten Max Imdahls formuliert: um die Identität des Bildes dar. ${ }^{38}$ In Frage stehen hierbei vor allem die Trennschärfe und damit die synthetisierende Kraft jenes Schnitts, dem zugestanden wird, die Differenz von bildlicher und nichtbildlicher Wirklichkeit definieren zu können. Dass eine kritische Auseinandersetzung mit dieser Modellierung von Bildlichkeit zuallererst eine Kritik der wenigstens seit Alberti metaphorisch geladenen Beschreibungssprache sein sollte, hat bereits André Bazin im Kontext seiner Versuche einer theoretischen Konzeptualisierung des Filmbildes in wünschenswerter Deutlichkeit angesprochen. Das von ihm gegenüber dem >cadre ins Spiel gebrachte und mit dem Begriff , cacher bezeichnete Modell vorläufiger Rahmung macht es möglich, das Verhältnis von Sichtbarkeit und Unsichtbarkeit nicht auf eine Konstellation von endgültig disjunkten Größen einschränken zu müssen. ${ }^{39}$ Bazins Konzept des scacher verweist auf jenes Moment dynamischer Entfaltung, das sich im Film fortlaufend als ein Zusammenspiel von On und Off ereignet, im einzelnen photographischen Bild indes als ein potenzielles Jenseits abzeichnet. Insbesondere

36 Siehe hierzu Philippe Junod: Vom "componimento inculto« Leonardos zum »œil sauvage» von André Breton. In: Gerhart von Graevenitz, Stefan Rieger, Felix Thürlemann (Hg.): Die Unvermeidlichkeit der Bilder, Tübingen 2001, S. 133-146. Dario Gamboni: Acheiropoiesis und potentielle Bilder im 19. Jahrhundert. In: Friedrich Weltzien (Hg.): von selbst. Autopoietische Verfahren in der Ästhetik des 19. Jahrhunderts, Berlin 2006, S. 63-74.

37 Hilde Zaloscer: Versuch einer Phänomenologie des Rahmens. In: Zeitschrift für Ästhetik und allgemeine Kunstwissenschaft 19 (1974), S. 189-224.

38 Max Imdahl: Überlegungen zur Identität des Bildes [1979]. In: ders.: Gesammelte Schriften, 3 Bde., Bd. 3, Frankfurt am Main 1996, S. 381-423. Siehe hierzu außerdem Max Imdahl: Bild - Totalität und Fragment. In: Lucien Dällenbach, Christiaan L. Hart Nibbrig (Hg.): Fragment und Totalität, Frankfurt am Main 1984, S. 115-123.

39 André Bazin: Peinture et cinéma. In: ders.: Qu’est-ce que le cinéma?, Paris ${ }^{11} 1999$, S. 187-192. Siehe hierzu auch Dubois 1990 (wie Anm. 4), S. 174-213 sowie den Beitrag von Ulrike Hanstein in diesem Band. 
von hier aus gewinnt die Rede vom `Silberspiegel ihren vollen Sinn: Dem statischen Ausschnitt der finestra aperta steht das Potenzial dynamischer Verwandlung eines scheinbar nur vorläufig definierten Ausschnittes entgegen. ${ }^{40}$

An den Rändern des photographischen Bildes ist das nicht Repräsentierte stets schon Gegenstand der Repräsentation. Es ist daher kein Zufall, dass in den hier näher betrachteten photographischen Collagen von David Hockney, Jan Wenzel und Peter Hendricks stets diese Ränder und die spezifische Gestaltung des Rahmens eine entscheidende Rolle spielen. Das ästhetische Kapital, das alle drei Künstler in jeweils sehr eigener Weise aus der Komposition einzelner Bilder zu einem größeren Ganzen schlagen, ist ohne die Optionen potenzieller photographischer Bildlichkeit undenkbar. Bei Hockney wird dieses Potenzial kubistisch verformt, um den Eindruck zeitlicher Dehnung in eine mal mehr, mal weniger homogene Bildfläche tragen zu können. Bei Wenzel ist die Latenz eines zwar unsichtbaren, aber dennoch präsenten bildlichen Jenseits der Anlass, Bildkonstruktionen von hoher Kohärenz zu entwerfen, deren ästhetische Prämisse jedoch gerade die Inkohärenz der visuellen Informationen ist. Bei Hendricks schließlich wird ein solcher, nicht zuletzt apparativ gestützter Ludismus in Tableaus übersetzt, deren analytischer Gestus zuletzt vor allem darauf zielt, das Verhältnis von Innerhalb und Außerhalb, von Sichtbarkeit und Unsichtbarkeit einer fortlaufenden Neubestimmung zu unterziehen und das Nebeneinander verschiedener Tafeln als den Wechsel von bildlicher Aktualität und Potenzialität sichtbar zu machen.

"Fotos", so meinte Susan Sontag mit Blick auf ein solches Zusammenspiel, "sind Details. Deshalb wirken Fotos so lebensähnlich. «41 Lebensähnlichkeit, heißt dies, ist ein Effekt, der mit den ästhetischen Mitteln des photographischen Bildes hergestellt werden kann. Und es ist nicht ohne Bedeutung, dass Sontag mit Blick auf solche Akte bildmedialer Produktion von 'Wirklichkeit eine Topik mobilisiert, deren Schlüsselbegriffe `Detail`, >Fragment` und `Bruchstück ‘ heißen. ${ }^{42}$ Sich für Fragen nach einer Bildästhetik des »Undsoweiter « zu interessieren, erfordert daher stets, den Blick auf die Funktion der Bildränder zu richten. Denn diese Ränder sind es, mit deren Hilfe sich das Zusammenspiel von Präsenz und Absenz, von Aktualität und Potenzialität überhaupt einrichten lässt. Sontags Identifikation des photographischen Bildes mit einer Ästhetik von Detail, Fragment und Bruchstück macht hierbei deutlich, dass zwischen den Operationen von Einschluss und Ausschluss stets ästhetische Praktiken des Anschlusses vermutet werden können. ${ }^{43}$

40 Für den originellen Versuch eines Ausgriffs auf dieses Jenseits des Bildes im Medium mittelalterlicher Buchmalerei siehe Felix Thürlemann: Die Miniatur und ihr Jenseits. Zu den Formaterweiterungen in den Très Riches Heures der Brüder Limburg. In: David Ganz, Thomas Lentes (Hg.): Ästhetik des Unsichtbaren. Bildtheorie und Bildgebrauch in der Vormoderne, Berlin 2004, S. 241-259.

41 Susan Sontag: Fotografie. Eine kleine Summa [2003]. In: dies.: Zur gleichen Zeit. Aufsätze und Reden, übers. von Reinhard Kaiser, München 2008, S. 164-167; hier S. 166.

42 Siehe hierfür ebd., insbesondere S. 164 und 166.

43 Mit einem vergleichenden Seitenblick auf die Malerei profilierte diesen Zusammenhang bereits pointiert Brian O'Doherty: In der weißen Zelle. Inside the White Cube [1976], übers. 
Das äußere Maß, das als Rahmen an die Photographie gelegt wird, ist gerade deshalb konstitutiv für jedes Interesse nach einer Ästhetik des photographischen Bildes, weil sich hier, anhand einer Vielfalt verschiedener Rahmenfunktionen, das Verhältnis von Teil und Ganzem organisiert. In Frage stehen damit zum einen die Optionen einer Transgression des photographischen Bildes, das heißt seiner Öffnung auf das, "was jenseits liegt«. Eine solche Frage zieht zum anderen aber nach sich, die "unendliche Mannigfaltigkeit", auf die die Transgression des photographischen Bildes zielt, in den Blick nehmen zu müssen. Die Idee von `Bezugnahme lässt sich auf diese Weise nicht allein als eine Frage des im Bild Sichtbaren diskutieren, sondern auch als eine Frage nach dem potenziellen photographischen Bild, das sich allein anhand ästhetischer Techniken der Produktion visueller Latenz abzeichnet. Dieses Jenseits jedoch mit ,Wirklichkeit` identifizieren zu wollen, arbeitet einer Vorstellung von photographischer Referenzialität zu, die sich in den Arbeiten von Hockney, Wenzel und Hendricks nachdrücklich dementiert sehen muss. Es war Susan Sontag, die einen Hinweis auf den Zielpunkt solcher Transgressionen formulierte: »Ein einzelnes Foto impliziert - anders als ein einzelnes Gemälde -, daß es noch andere gibt. ${ }^{44}$ Fragen nach einer Ästhetik des potenziellen photographischen Bildes führen notwendigerweise zu einer Betrachtung des Bildes im Plural. Denn an den Rändern jedes einzelnen Bildes, wie scharf oder unscharf seine Grenzen auch gezogen sind, lauern stets neue Bilder.

von Wolfgang Kemp, Berlin 1996, S. 13-17. Siehe außerdem Oskar Bätschmann: Begrenzt - Unbegrenzt. In: Werner Busch, Oliver Jehle (Hg.): Vermessen. Landschaft und Ungegenständlichkeit, Zürich, Berlin 2007, S. 57-72.

44 Susan Sontag: Die Bilderwelt. In: dies.: Über Fotografie, [New York 1977], übers. von Mark W. Rien und Gertrud Baruch, München, Wien 1978, S. 141-166; hier S. 153. 